\title{
Long-term follow-up of extended aortoplasty for supravalvular aortic stenosis
}

Extended aortoplasty is an operation that was designed to provide a symmetric reconstruction of the aortic root in patients with supravalvular aortic stenosis. The aim of this report is to provide long-term follow-up of the original cohort of 15 patients who underwent extended aortoplasty between 1975 and 1983. Follow-up was obtained in 14 patients. One patient was lost to follow-up 3 years after operation; he was included in this report. An echocardiogram, chest radiograph, and electrocardiogram were obtained for each surviving patient. The median length of follow-up was 141 months (range 36 to 238). The median preoperative gradient was $90 \mathrm{~mm} \mathrm{Hg}$ (range 55 to 150). The median immediate postoperative gradient was $20 \mathrm{~mm} \mathrm{Hg}$ (range 0 to 50, $p<0.05$ compared with preoperative gradient) and the median long-term gradient was $32 \mathrm{~mm} \mathrm{Hg}$ (range 6 to 96, $p<0.05$ compared with preoperative gradient; $p=$ not significant compared with immediate postoperative gradient). Two patients died: one of left ventricular failure after a subsequent aortic valve replacement and one of chronic left ventricular failure. The Kaplan-Meier estimate of survival at 218 months for all patients was $77.4 \%$ ( $70 \%$ confidence limits $62 \%$ to $93 \%$ ). The estimated freedom from reoperation for all patients was $69 \%$ at 218 months $(70 \%$ confidence limits $56 \%$ to $82 \%$ ). Univariate analysis revealed that the presence of a bicuspid valve is a significant risk factor for reoperation $(p=0.038)$, but not for death $(p=0.51)$. The Kaplan-Meier estimate of freedom from reoperation for patients with a bicuspid aortic valve was $42.9 \%$ at 141 months (70\% confidence limits $21 \%$ to $65 \%$ ). Extended aortoplasty provides effective long-term relief of the pressure gradient across the supravalvular ridge. However, a significant number of patients require subsequent operations, particularly those with a bicuspid aortic valve. (J THORAC CARDIOVASC SURG 1995;109:155-63)

Ralph E. Delius, $\mathrm{MD}^{\mathrm{a}}$ (by invitation), John B. Steinberg, $\mathrm{MD}^{\mathrm{a}}$ (by invitation), Thomas L'Ecuyer, $\mathrm{MD}^{\mathrm{b}}$ (by invitation), Donald B. Doty, $\mathrm{MD},{ }^{\mathrm{c}}$ and Douglas M. Behrendt, MD, ${ }^{a}$ Iowa City, Iowa, and Salt Lake City, Utah

$\mathrm{S}_{\mathrm{u}}$ congenital cause of left ventricular outflow obstruction. Classically, three morphologic subtypes have been described: an hourglass type, a membranous type, and a diffuse type. ${ }^{1}$ Clinically, the hourglass and membranous types are managed similarly. The

From the Divisions of Cardiothoracic Surgery and Pediatric Cardiology, ${ }^{\mathrm{b}}$ University of Iowa Hospitals and Clinics, Iowa City, Iowa, and the Division of Cardiothoracic Surgery, ${ }^{c}$ LDS Hospital, Salt Lake City, Utah.

Read at the Seventy-fourth Annual Meeting of The American Association for Thoracic Surgery, New York, N.Y., April 24-27, 1994.

Address for reprints: Douglas M. Behrendt, MD, 1602-A John Colloton Pavillion, University of Iowa Hospitals and Clinics, Iowa City, LA 52242.

Copyright (c) 1995 by Mosby-Year Book, Inc.

$0022-5223 / 95 \$ 3.00+0 \quad \mathbf{1 2 / 6 / 6 0 3 3 4}$ diffuse subtype, which involves hypoplasia of a variable length of ascending aorta, often necessitates different surgical approaches.

Although this lesion was first described by Cheevers in $1842,{ }^{2}$ it was first fully characterized in 1959 by Morrow and colleagues ${ }^{3}$ at the National Institutes of Health. Shortly thereafter McGoon and associates ${ }^{4}$ at the Mayo Clinic reported the surgical correction of supravalvular aortic stenosis in a series of patients. This surgical repair involved placement of a teardrop-shaped patch across the supravalvular ring. The short-term results of this operation were acceptable and it remained the procedure of choice for a number of years. However, a report by Keane and associates 5 noted that a significant portion $(>50 \%)$ of patients had a gradient greater than 30 $\mathrm{mm} \mathrm{Hg}$ at long-term follow-up. Flaker and colleagues ${ }^{6}$ also reported that significant residual ab- 
Table I. Associated cardiac diagnoses

\begin{tabular}{lc}
\hline \multicolumn{1}{c}{ Diagnosis } & $\begin{array}{c}\text { No. of } \\
\text { patients }\end{array}$ \\
\hline Bicuspid aortic valve & 6 \\
Bicuspid aortic valve with coarctation, & 1 \\
$\quad$ ventricular septal defect, pulmonary & \\
artery stenosis & 3 \\
No other abnormalities & 2 \\
Pulmonary artery stenosis & 1 \\
Subaortic stenosis & 1 \\
Subaortic stenosis with ventricular & \\
$\quad$ septal defect & 1 \\
Subaortic stenosis with peripheral & \\
pulmonary artery stenosis & $\overline{15}$ \\
Total &
\end{tabular}

normalities such as excessive gradients and aortic insufficiency were relatively frequent after simple patch aortoplasty.

Extended aortoplasty, first described by Doty, Polansky, and Jensen ${ }^{7}$ in 1975, was designed in response to a patient who had a significant postoperative gradient and severe hemolytic anemia after a simple patch aortoplasty. This operation involves placement of an inverted $\mathrm{Y}$-shaped incision across the supravalvular constricting ring and into the right and noncoronary sinuses of Valsalva, followed by insertion of a pantaloon-shaped Dacron fabric patch. The operation was designed to provide a more symmetric reconstruction of the aortic root than that obtained with a simple patch. Extended aortoplasty has been widely adopted since the original description, ${ }^{8,9}$ although there have been few follow-up reports of this operation since its initial description. The aim of this report is to provide long-term follow-up of the initial group of patients who underwent extended aortoplasty for supravalvular aortic stenosis.

\section{Methods}

Patient population. Fifteen patients with supravalvular aortic stenosis underwent extended aortoplasty between June 1975 and December 1983. Twelve patients were male and three were female. All operations were done by a single surgeon (D. D.). Two patients had William's syndrome, 2 patients who were siblings had a familial history of supravalvular aortic stenosis, and 11 patients had the sporadic form. One patient had diffuse supravalvular stenosis and the remainder had the discrete form. Associated cardiac diagnoses are shown in Table I. Preoperative symptoms included exercise intolerance in seven patients, angina in two patients, and syncope in one patient. Five patients were free of symptoms before operation. The diagnosis and pressure gradients were established by cardiac catheterization preoperatively in all patients. Postoperative peak gradients were measured in the operating room after the patient was weaned from cardiopulmonary bypass by direct needle puncture. Peak gradients were determined at long-term follow-up by continuous wave Doppler flow analysis. In addition, four patients also underwent late cardiac catheterization. In these patients there was excellent correlation (less than $15 \%$ variation) between peak gradients determined by catheterization and those determined by continuous wave Doppler flow analysis.

The median age at the time of operation was 6.5 years, with a range of 3.5 to 15 years. All patients were discharged from the hospital alive. The only in-hospital complication was a single case of postpericardiotomy syndrome, which resolved after a brief course of a nonsteroidal antiinflammatory agent.

Complete follow-up to January 1, 1994, was obtained in 14 of the 15 patients. One patient was lost to follow-up after 36 months; he was included in this report. The charts of all patients were reviewed. Patients who had not been followed up within the past year were contacted and invited to come to Iowa City or an outreach clinic for a complete examination, including a chest radiograph, electrocardiogram, and echocardiogram. Patients who had moved from the local area and were being followed up by a local cardiologist had copies of their medical records sent, including results of the latest electrocardiogram, chest radiograph, and echocardiogram. When necessary, medical records were updated by telephone contact. Permission to contact patients was obtained from the University of Iowa Institutional Review Board.

Operative technique. After median sternotomy and heparinization, cardiopulmonary bypass was initiated by means of a single venous cannula and an arterial cannula placed in the ascending aorta near the takeoff of the brachiocephalic artery. All patients had a left ventricular vent placed. Crystalloid cardioplegia was administered after application of the aortic crossclamp. An inverted $Y$-shaped incision was then made in the ascending aorta, with the two arms of the Y extending across the supravalvular ring into the noncoronary and right sinuses of Valsalva. The incisions across the fibrosing ring were approximately 180 degrees apart. The incision into the right coronary sinus was placed to the left side of the right coronary ostia. The fibrous ring was then excised from above the left coronary sinus. In two patients with a bicuspid valve a commissurotomy was done. A Dacron fabric graft approximately the diameter of the ascending aorta was divided longitudinally and then cut to length. A wedge was taken from one end of the graft to accommodate the area of aorta around the right coronary ostia, giving a pantaloon shape to the patch. The patch was then secured in place with monofilament suture. The final appearance of the ascending aorta can be seen in Fig. 1.

Statistics. Fisher's exact test was used to determine associations between preoperative variables and death or reoperation. Kaplan-Meier estimates were obtained for long-term survival and freedom from reoperation. Risk factors for death and reoperation were assessed by univariate analysis and forward stepwise multivariate analysis by Wilcoxon and log-rank tests. The association between postoperative and long-term gradients was examined by linear regression. Student's $t$ test was used to test the 
difference in gradients in patients with and without left ventricular hypertrophy. For each test a $p$ value equal to or less than 0.05 was considered significant.

\section{Results}

Hemodynamics. The median and mean preoperative gradients were 90 and $91 \mathrm{~mm} \mathrm{Hg}$, respectively, with a range of 55 to $150 \mathrm{~mm} \mathrm{Hg}$. The median and mean postoperative gradients were 20 and $23 \mathrm{~mm} \mathrm{Hg}$, respectively, with a range of 0 to $50 \mathrm{~mm} \mathrm{Hg}$. The median and mean long-term gradients were 32 and 36 $\mathrm{mm} \mathrm{Hg}$, respectively (range 6 to $96 \mathrm{~mm} \mathrm{Hg}$ ) (Fig. 2).

As expected, the mean preoperative gradient was significantly greater than the postoperative gradient $(p=0.01)$. There was no statistical difference between the postoperative and long-term gradients. However, linear regression analysis revealed that there was no clear correlation between the postoperative and long-term gradients (Fig. 3). Thus the immediate postoperative gradient was found to be a poor predictor of the long-term gradient. This could have been because of the different techniques used to measure the two gradients (direct measurement versus continuous wave Doppler flow analysis), progressive stenosis at the supravalvular ring or another level of the left ventricular outflow tract, or varying hemodynamic conditions (such as cardiac output, afterload) at the time of the two measurements.

Five patients with significant long-term gradients $(\geq 40 \mathrm{~mm} \mathrm{Hg}$ ) who underwent reoperation were found to have obstruction at the level of the aortic valve rather than at the supravalvular ring. The two other patients with long-term gradients $40 \mathrm{~mm} \mathrm{Hg}$ or higher were found to have normal aortic root dimensions and normal supravalvular flow velocities, which also suggests that the level of the gradient was not at the level of the supravalvular ring.

Six patients $(40 \%)$ had preoperative aortic insufficiency; one characterized as mild, the remainder as moderate. Five of these six patients had persistent aortic insufficiency at long-term follow-up. Aortic insufficiency was found to have developed in four additional patients during the period of follow-up, giving an incidence at follow-up of $60 \%$ (9/15). Four of these patients had aortic insufficiency characterized as mild, three as moderate, and two as severe.

All patients had preoperative left ventricular hypertrophy shown by electrocardiogram. At longterm follow-up nine patients $(60 \%)$ had persistent left ventricular hypertrophy. Seven of these $(78 \%)$ had gradients $40 \mathrm{~mm} \mathrm{Hg}$ or higher. All patients with gradients $40 \mathrm{~mm} \mathrm{Hg}$ or higher demonstrated electrocardiographic evidence of left ventricular hyper-

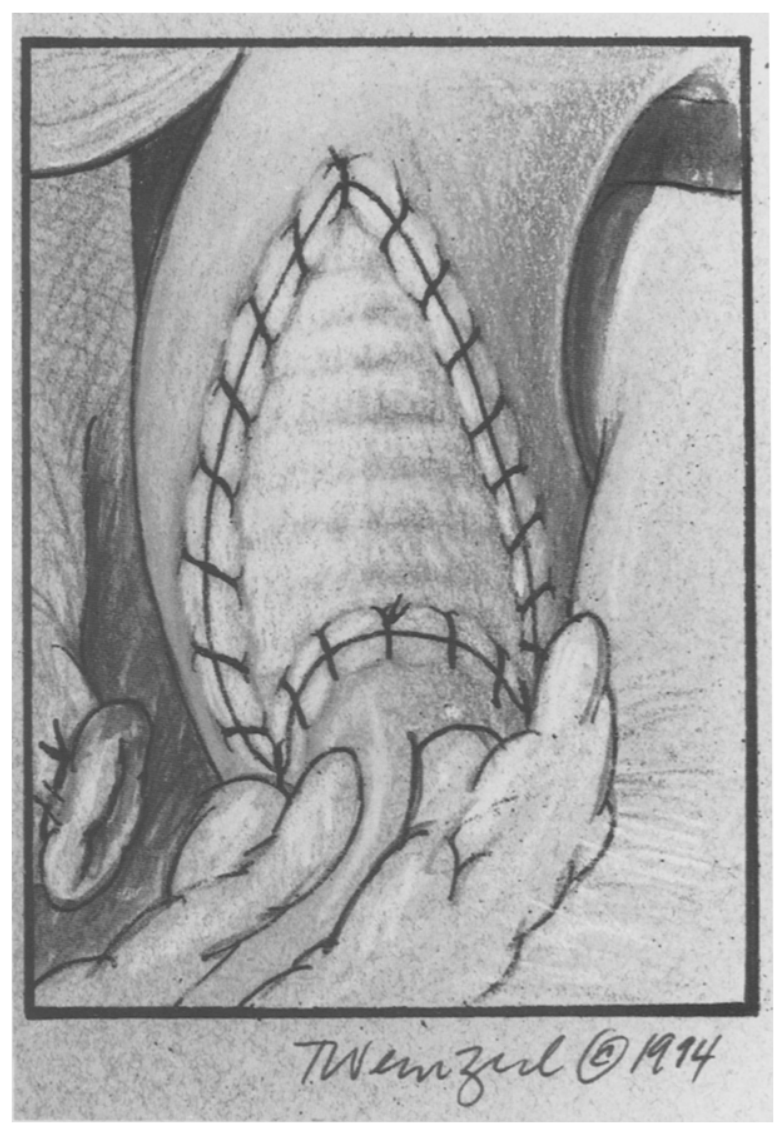

Fig. 1. Final appearance of ascending aorta after extended aortoplasty. Note that patch extends into right and noncoronary sinuses of Valsalva.

trophy (Fig. 4). The sensitivity of electrocardiographic evidence of left ventricular hypertrophy for a gradient $40 \mathrm{~mm} \mathrm{Hg}$ or higher was $100 \%$ and the specificity $75 \%$.

The single patient with the diffuse type of supravalvular aortic stenosis had the aortoplasty patch extended distally to the takeoff of the brachiocephalic artery. A cardiac catheterization done 4 months after operation showed that the residual gradient gradually became more pronounced as the ascending aorta and aortic arch were traversed rather than occurring at a single point. At long-term follow-up this patient had a peak gradient of $50 \mathrm{~mm}$ $\mathrm{Hg}$ at the level of the aortic valve by continuous wave Doppler flow analysis. A subsequent catheterization confirmed the gradient across the aortic valve $(42 \mathrm{~mm} \mathrm{Hg}$ ) but did not demonstrate any distal aortic gradient.

Reoperation. Eight reoperations were done in six patients. The reoperations done and the indications for reoperation are shown in Table II. Only one 


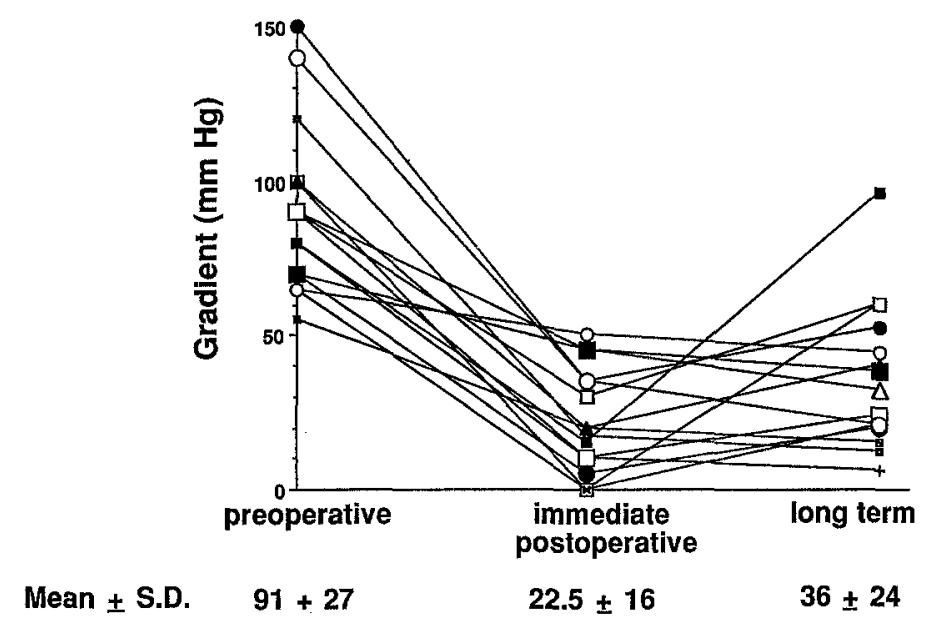

Fig. 2. Preoperative, postoperative, and long-term peak gradients. Preoperative gradients were determined by cardiac catheterization, postoperative gradients by direct measure in operating room, and long-term gradients by continuous wave Doppler flow analysis $(p=0.01$ preoperative versus postoperative or long-term gradients; $p=$ not significant postoperative versus long-term gradients). S.D., Standard deviation.

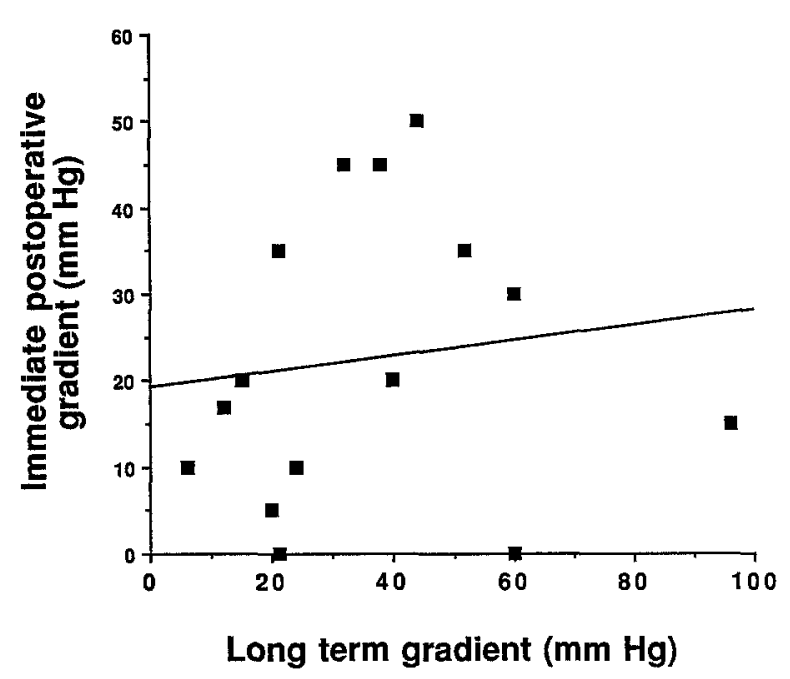

Fig. 3. Linear regression analysis of postoperative versus long-term peak gradients $\left(r^{2}=0.016, p=\right.$ not significant).

reoperation was directly related to supravalvular stenosis or extended aortoplasty. This patient was having transient ischemic attacks after operation. Incomplete neointimal lining of the Dacron fabric patch was postulated as the source of emboli. The original patch was replaced with a larger, knitted velour Dacron fabric patch. No clear source for the emboli was identified. The remainder of the reoperations were to alleviate aortic valve pathologic conditions rather than the supravalvular stenosis.

The Kaplan-Meier estimate of freedom from reoperation for all patients was 69\% (confidence limits [CL] 56\% to $82 \%$ ) at 216 months (Fig. 5). Univariate analysis revealed that the presence of a bicuspid aortic valve $(p=0.03)$ and a long-term gradient $40 \mathrm{~mm} \mathrm{Hg}$ or higher $(p=0.04)$ were associated with an increased risk of reoperation. Forward stepwise multivariate analysis revealed that only the presence of a bicuspid aortic valve was a risk factor for reoperation $(p=0.03)$. The KaplanMeier estimate of freedom from reoperation for patients with a bicuspid aortic valve was $42.9 \%$ (CL $21 \%$ to $65 \%$ ) at 149 months, whereas freedom from reoperation for patients with a tricuspid aortic valve was $87.5 \%$ (CL 76\% to 99\%) at 216 months (Fig. 6).

Survival. Two patients died during the follow-up period. The Kaplan-Meier estimate of survival at 216 months (18 years) was $77.4 \%$ (70\% CL $62 \%$ to 93\%) (Fig. 7). One patient died 169 months after operation of heart failure while awaiting a heartlung transplant. This patient also had subvalvular stenosis, pulmonary stenosis, and a ventricular septal defect. Another patient died 89 months after operation of heart failure after valve replacement for aortic insufficiency. Reoperation $(p=0.01)$ and a long-term gradient $40 \mathrm{~mm} \mathrm{Hg}$ or higher $(p=0.04)$ were independently associated with an increased risk of death. No other risk factors were identified. With only two deaths in this report, however, these associations do not have much statistical power.

Symptoms and functional status. Eleven of the 12 remaining patients ( 2 deaths, 1 lost to follow-up) are free of symptoms (class I). One patient has mild restrictions because of exercise intolerance (class 


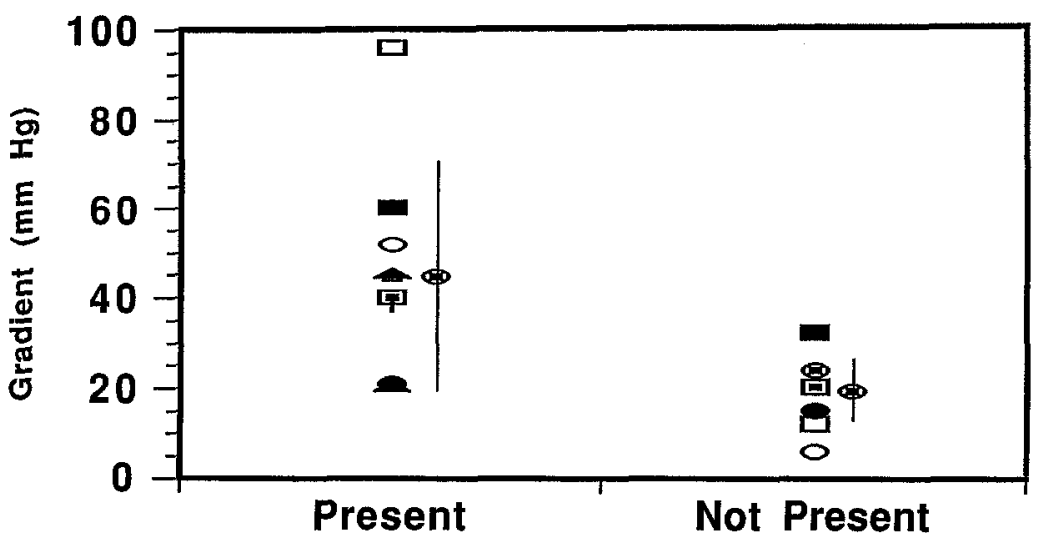

\section{LVH}

Fig. 4. Distribution of peak gradients in patients with electrocardiographic evidence of left ventricular hypertrophy $(L V H)$ (gradient if left ventricular hypertrophy present versus gradient if left ventricular hypertrophy not present, $p=0.02$ ). Error bars indicate standard deviation.

II). One patient had a normal pregnancy. Eleven patients are employed or in school. One patient is not employed, but the reason for unemployment is unrelated to health or functional status.

\section{Discussion}

At first glance supravalvular aortic stenosis appears to be a relatively simple lesion. Attention usually focuses on the constricting supravalvular ring. However, supravalvular aortic stenosis is actually a complex lesion that often involves the entire left ventricular outflow tract. The primary abnormality involves intimal hyperplasia and thickening of the aortic media immediately above the sinuses of Valsalva, which results in narrowing of the aorta. The presence of this fibrosing ring can have many secondary effects. The sinuses themselves may be hypoplastic. The stenosing ring often affects the relationships of the commissures, which leads to distortion of the aortic cusps, particularly the free edges. ${ }^{3}$ Occasionally, the free edges of the cusp may even fuse to the aortic wall, effectively isolating the sinus of Valsalva and coronary ostia from the lumen of the aorta. ${ }^{1}$ A significant number of patients also have dysplasia and irregular fibrous thickening of the aortic cusps. ${ }^{10}$

The coronary arteries are also often dilated and injured by being exposed to the elevated pressures that are present proximal to the supravalvular ridge. One report noted significant coronary artery abnormalities in $49 \%$ of patients, with thickening of the intima and media noted as early as 3 years of age. ${ }^{11}$ All older patients in the same report had extensive coronary artery disease, with evidence of prior in-
Table II. Reoperations

\begin{tabular}{cllr}
$\begin{array}{c}\text { Patient } \\
\text { No. }\end{array}$ & \multicolumn{1}{c}{ Reoperation done } & Indication & $\begin{array}{c}\text { Time to } \\
\text { reoperation } \\
\text { (mo) }\end{array}$ \\
\hline 1 & Aortic root replacement & AS & 238 \\
2 & (1) Replace patch & TIA & 2 \\
& (2) AVR & AS/AI & 2 \\
3 & AVR & AS & 37 \\
4 & AVR & AS/AI & 139 \\
5 & AVR & AS/AI & 143 \\
6 & (1) Aortic valvuloplasty & AI & 19 \\
& (2) Aortic root replacement & AS/AI & 165 \\
\hline
\end{tabular}

$A S$, Aortic stenosis; $T I A$, transient ischemic attack; $A V R$, aortic valve replacement; $A I$, aortic insufficiency.

farctions. In some instances the coronary ostia may be narrowed by the fibrosing ring and may even become obstructed entirely if the aortic cusp fuses to the aortic wall. ${ }^{1}$

In addition to the abnormalities secondarily related to the supravalvular stenosis, associated lesions of the left ventricular outflow tract are also relatively common. In this report $47 \%$ of the patients had a bicuspid aortic valve. Subvalvular stenosis also occurs. Pulmonary artery stenosis and other abnormalities are often found in patients with William's syndrome.

Multiple operations have been described to relieve supravalvular aortic stenosis, including manual dilation of the fibrotic ring, ${ }^{12}$ resection of the fibrosing ring with reanastomosis of the aorta, ${ }^{13,14}$ and the use of an apicoaortic conduit in particularly difficult cases. ${ }^{15}$ The most common operations currently done include patch aortoplasty, primary reconstruction of the sinuses of Valsalva, and ex- 


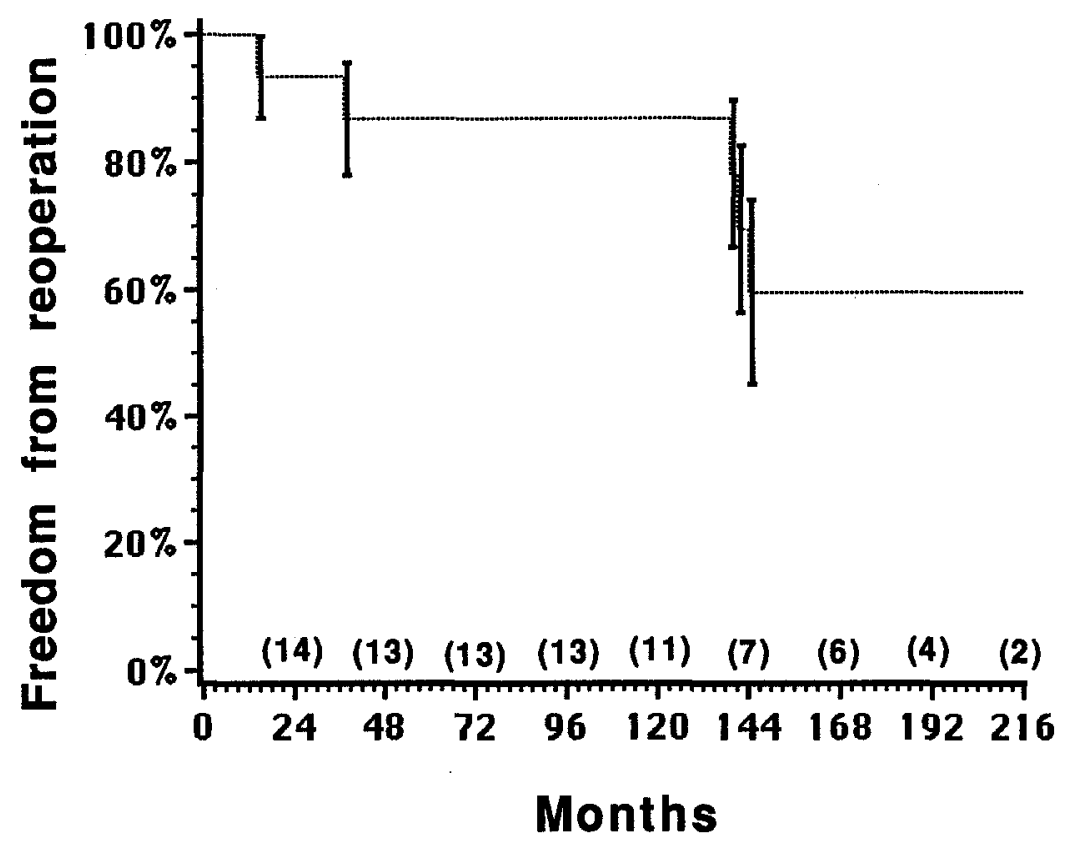

Fig. 5. Kaplan-Meier estimate of freedom from reoperation. Error bars indicate $70 \%$ CL. Numbers of patients at risk are indicated in parentheses.

tended aortoplasty. Patch aortoplasty, first described by McGoon and associates ${ }^{4}$ in 1961, involves excision of the supravalvular ring and placement of a teardrop-shaped patch across the ring in the noncoronary sinus of Valsalva. The long-term results of this operation have been mixed. Long-term follow-up of the Mayo Clinic experience with patch aortoplasty has recently been reported, with good long-term relief of the supravalvular gradient in patients with discrete supravalvular aortic stenosis. ${ }^{11}$ A follow-up study from the Texas Heart Institute reported good long-term clinical results with patch aortoplasty, but no hemodynamic data were provided. ${ }^{16}$ However, other long-term follow-up studies of patch aortoplasty have reported persistent gradients, which were often believed to be related to the ring rather than other lesions of the left ventricular outflow tract. ${ }^{5,6}$

Extended aortoplasty was designed to address the theoretic shortcomings of patch aortoplasty, in particular the asymmetric reconstruction of the aortic root. $^{7}$ By dividing the supravalvular ring at two sites approximately 180 degrees apart, the right and noncoronary commissures acquire a more normal relationship, which in turn allows the corresponding cusps to regain their normal geometry. Debridement of the rim above the left sinus of Valsalva may allow the left cusp to assume a normal position, but in some cases an incision into the left sinus and patch repair may be required before the left cusp can be normally positioned. Symmetric opening of the supravalvular ring and restoration of cusp anatomy ensures wide opening of the aortic root and relieves potential obstruction of the coronary ostia.

Recently, direct repair of each of the sinuses of Valsalva with either prosthetic patches or flaps of the ascending aorta has been described. ${ }^{17}$ This approach appears to best restore the normal anatomic relationships of the aortic valve and root. In addition, use of the aortic flaps offers the advantage of incorporating only autologous tissue, which may provide better potential for growth. However, longterm clinical and hemodynamic follow-up is not yet available for these procedures.

In this and other reports, the primary source of morbidity and mortality appears to be related to associated aortic valve pathologic conditions. In the Texas Heart Institute series, $81 \%$ of the reoperations and all of the late deaths could be attributed to aortic valve dysfunction or infection. ${ }^{16}$ The Mayo Clinic study reported that an aortic valve pathologic condition was the only identifiable predictor of late death or reoperation by multivariate analysis. ${ }^{11}$ In the present report all but one of the reoperations could be attributed to aortic 


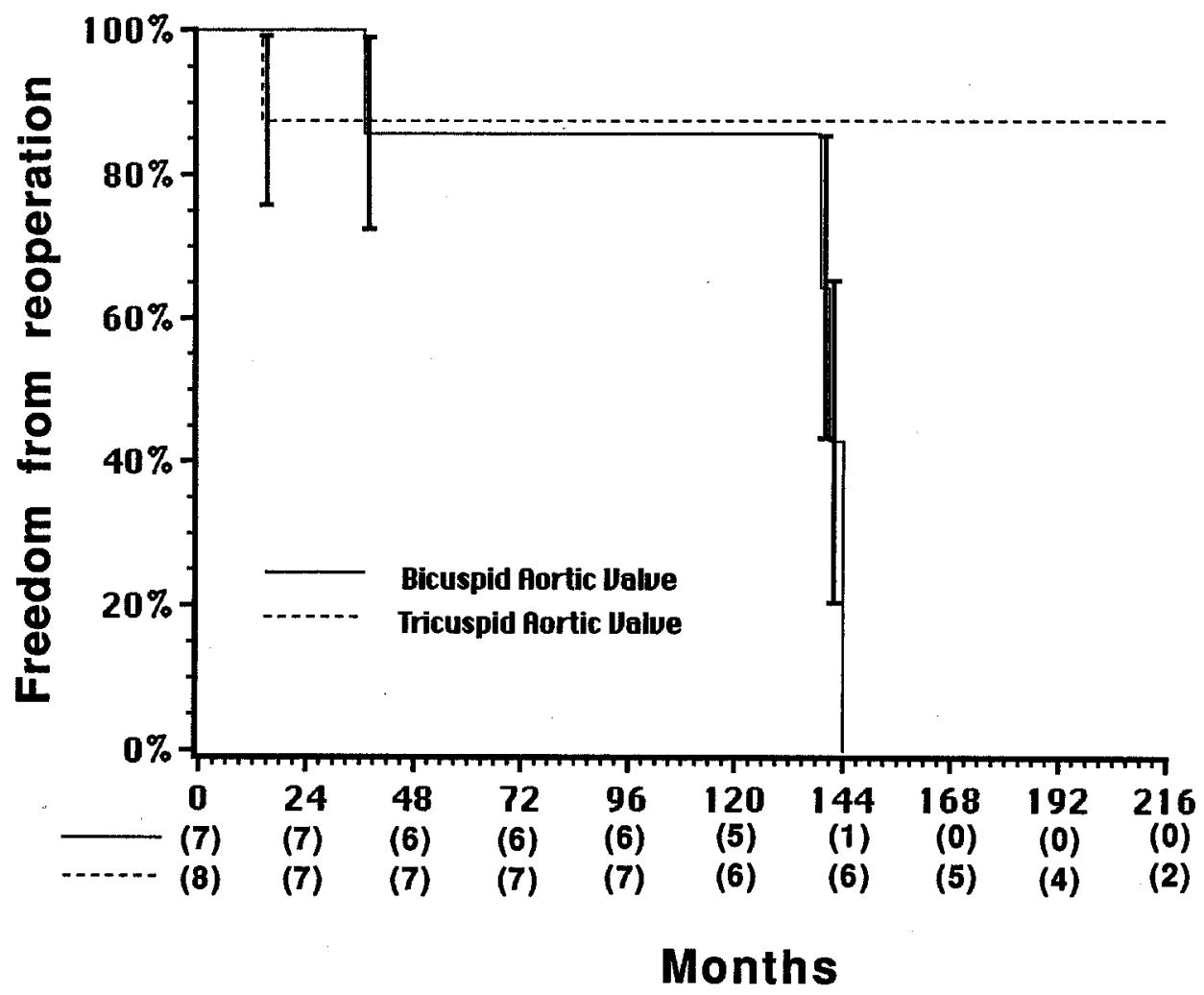

Fig. 6. Kaplan-Meier estimates of freedom from reoperation for patients with bicuspid or tricuspid aortic valve $(p=0.03)$. Error bars indicate $70 \%$ CL. Numbers of patients at risk are indicated in parentheses.

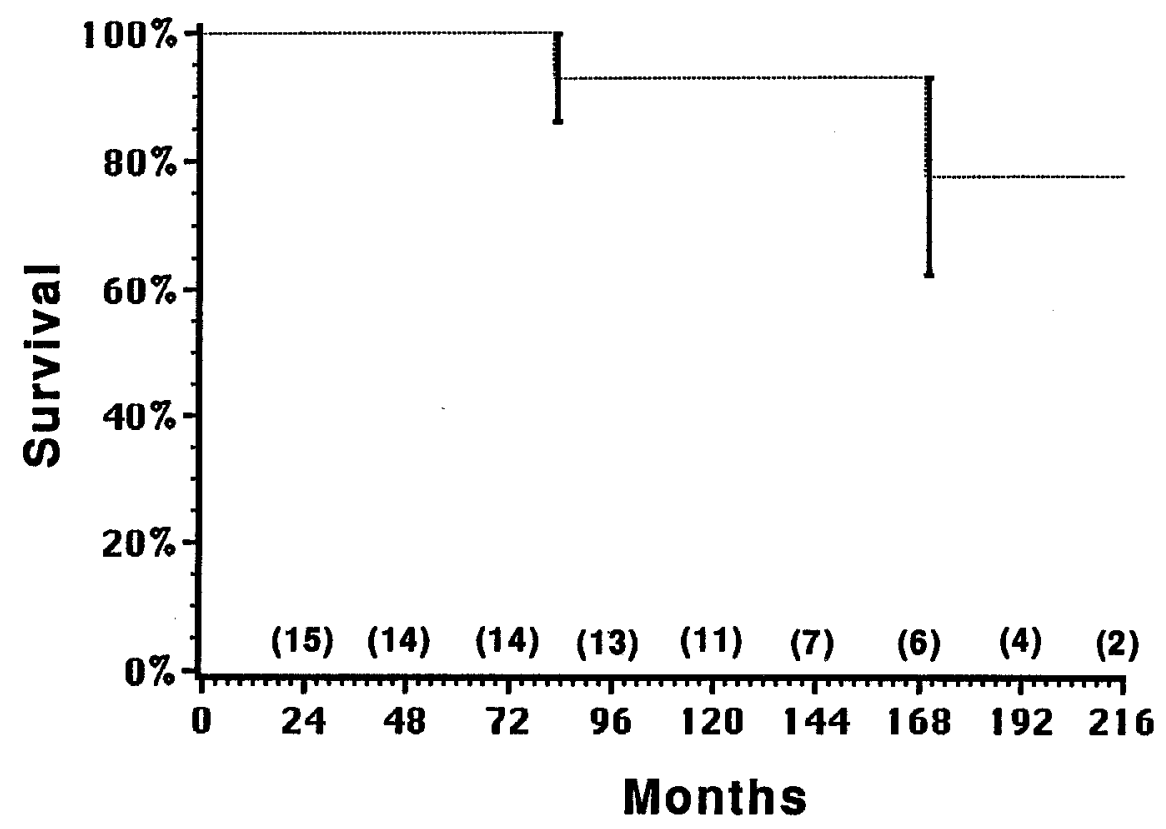

Fig. 7. Kaplan-Meier estimate of survival. Error bars indicate $70 \%$ CL. Numbers of patients at risk are indicated in parentheses. 
valve abnormalities rather than the supravalvular ring. In particular, the presence of a bicuspid valve, which is often associated with an elevated gradient, appears to be a significant risk factor for reoperation. Persistent left ventricular hypertrophy appears to be a sensitive marker for gradients greater than $40 \mathrm{~mm} \mathrm{Hg}$, although all of these patients are followed up by serial echocardiograms and any increased gradients would also be detected by this technique.

The one reoperation that could be attributed directly to extended aortoplasty was replacement of a patch because of transient ischemic attacks. The concern before reoperation was that the Dacron patch had not developed a neointimal lining, leaving a thrombogenic surface. At operation no clear source of emboli was identified. We have subsequently used polytetrafluoroethylene for the patch without any complications. The Mayo Clinic group advocates the use of autologous pericardium for the patch. ${ }^{18}$ They report no late aneurysm formation or other complications related to this patch. The use of autologous pericardium or polytetrafluoroethylene can be easily adapted to extended aortoplasty and may be preferable to the use of Dacron fabric.

Supravalvular aortic stenosis is a rare, complex abnormality of the left ventricular outflow tract. Extended aortoplasty appears to be an effective, albeit palliative, operation for discrete supravalvular aortic stenosis. A good hemodynamic result across the supravalvular ring, however, does not ensure against reoperation. Reoperation is common, but appears to be related to associated lesions of the left ventricular outflow tract rather than to the supravalvular stenosis.

We thank C. Kice Brown, MS, for statistical consultation and Thomas Weinzerl, MA, and Darrin Pigg, MA, for illustrations and graphs.

\section{REFERENCES}

1. Peterson TA, Todd DB, Edwards JE. Supravalvular aortic stenosis. J Thorac CARdIOvasc SuRg 1965;50: 734-41.

2. D'Cruz IA, Miller RA. Norman Cheevers: a description of the congenital absence of pulmonary valves and supravalvular aortic stenosis in the $1840 \mathrm{~s}$. Br Heart J 1964;26:723-5.

3. Morrow AG, Waldhausen JA, Peters RL, Bloodwell RD, Braunwald E. Supravalvular aortic stenosis: clinical, hemodynamic and pathologic observations. Circulation 1959;20:1003-10.

4. McGoon DC, Mankin HT, Vlad P, Kirklin JW. The surgical treatment of supravalvular aortic stenosis. $\mathbf{J}$ Thorac CARdiovasC Surg 1961;41:125-33.

5. Keane JF, Fellows KE, LaFarge CG, Nadas AS, Bernhard WF. The surgical management of discrete and diffuse supravalvar aortic stenosis. Circulation 1976;54:112-7.

6. Flaker G, Teske D, Kilman J, Hosier D, Wooley C. Supravalvar aortic stenosis: a 20 year clinical perspective and experience with patch aortoplasty. Am J Cardiol 1983;51:256-60.

7. Doty DB, Polansky DB, Jensen CB. Supravalvular aortic stenosis: repair by extended aortoplasty. $\mathrm{J}$ THORAC CARDIOVASC SURG 1977;74:362-71.

8. Graham JM, Lawrie GM, Eid Fawzy M, DeBakey ME. Severe supravalvular stenosis: successful surgical correction by extended aortoplasty. Ann Thorac Surg 1982;34:698-701.

9. Stewart S, Alexson C, Manning J. Extended aortoplasty to relieve supravalvular aortic stenosis. Ann Thorac Surg 1988;46:427-9.

10. Williams JCP, Barratt-Boyes BG, Lowe J. Supravalvular aortic stenosis. Circulation 1961;24:1311-8.

11. van Son JAM, Danielson GK, Puga FJ, et al. Supravalvular aortic stenosis: long term results of surgical treatment. J ThORAC Cardiovasc Surg 1994;107:103-15.

12. Kreel I, Reiss R, Strauss L, Blumenthall S, Baronofsky ID. Supravalvar stenosis of the aorta. Ann Surg 1959;149:519-24.

13. Hara M, Dungan T, Lincoln B. Supravalvular aortic stenosis: report of successful excision and aortic reanastomosis. J Thorac CARDIovasc SuRg 1962;43: 212-21.

14. Chard RB, Cartmill TB. Localized supravalvar aortic stenosis: a new technique for repair. Ann Thorac Surg 1993;55:782-4.

15. Sweeney MS, Walker WE, Cooley DA, Reul G. Apicoaortic conduits for complex left ventricular outflow obstruction: 10 year experience. Ann Thorac Surg 1986;42:609-11.

16. Sharma BK, Fujiwara H, Hallman GL, Ott DA, Reul GJ, Cooley DA. Supravalvar aortic stenosis: a 29 year review of surgical experience. Ann Thorac Surg 1991; 51:1031-9.

17. Myers JL, Waldhausen JA, Cyran SE, Gleason MM, Weber HS, Baylen BG. Results of surgical repair of congenital supravalvular aortic stenosis. J THORAC Cardiovasc Surg 1993;105:281-8.

18. Piehler JM, Danielson GK, Pluth JR, et al. Enlargement of the aortic root or annulus with autogenous pericardial patch during aortic valve replacement. J Thorac Cardiovasc Surg 1983;86:350-8.

\section{Discussion}

Dr. John L. Myers (Hershey, Pa.). I congratulate Dr. Delius on a nice presentation and all of the authors for an important paper detailing the 10- to 20-year follow-up on 
the extended pantaloon patch technique described by Dr. Doty in a series of his original patients. There is no doubt that this technique can achieve excellent gradient reduction in the supravalvular region, as demonstrated in these patients and in the results that have been demonstrated by other groups, and with good patient survival.

However, we would hypothesize that one of the causes for the high incidence of reoperation may be related to two specific factors: one, the later date of initial repair and thus a longer period of exposure to the abnormal hemodynamics, which can cause additional damage to the aortic valve leaflets and coronary ostia, and two, this technique does not necessarily result in complete restoration of normal geometry of the aortic root.

Our philosophy has been toward earlier and earlier repair when we see these patients, proceeding to operation before the development of left ventricular hypertrophy and, we hope, before the development of aortic valve leaflet damage. We have used a technique, which was modified from an original technique by Dr. Brahm, that restores normal aortic root geometry by enlarging the aorta in all three sinuses of Valsalva and, whenever possible, uses distal aortic tissue.

We presented our original paper 2 years ago at this Association. These patients had a mean age of $4 \frac{1}{2}$ years and a median age of 2 years. The follow-up period is now $3 \frac{1}{2}$ years and ranges from 1 to 5 years. By two-dimensional Doppler echocardiography, which is clearly oversensitive, and certainly in your results the slight increase in gradient may be just reflective of the oversensitivity of echocardiography, there is a mean gradient of $10 \mathrm{~mm} \mathrm{Hg}$ with no change in follow-up, which albeit is quite short. None of our patients has left ventricular hypertrophy. We evaluated the patients for residual insufficiency and found trace or mild insufficiency by Doppler echocardiography in four patients, but no diastolic murmurs that were audible.
Could you tell from your analysis whether any of these six patients who had reoperation for aortic valve replacement had evidence of significant aortic valve pathologic conditions at the original operation? In other words, are these developments of aortic stenosis and aortic insufficiency merely a propagation of aortic valve damage that might be avoided by operating much earlier in the course of the disease?

Dr. Delius. I was thankful that Dr. Doty dictated meticulous operative notes, and he noted on multiple occasions that although the cusps were basically normal, they appeared somewhat thickened. Therefore I think the idea of early operation has a lot of merit. Our patients were usually operated on at about 5 or 6 years old, which was the custom in the late 1970 s, but I think the idea of operating on the patients at an earlier date is an interesting one that may have some merit.

Dr. Jacques A. M. van Son (San Francisco, Calif.). Were autopsies done in the two patients who died and was there any evidence of coronary artery disease or myocardial fibrosis? We reported a surgical series of 80 patients from the Mayo Clinic, in which six of the eight patients who died had autopsy-proven coronary artery disease and myocardial fibrosis. A subsequent pathologic study of five hearts with supravalvular aortic stenosis identified the extraaortic content of this condition including dissection of the ascending aorta and the development of ischemic heart disease as early as early childhood. On the basis of these findings I agree with Dr. Meyers that operation for supravalvular aortic stenosis should be done as early in life as possible.

Dr. Delius. We did not detect any significant coronary artery disease, although I know in one patient it was noted that the coronary arteries were rather thick, which was consistent with what you reported last year. However, none of the patients in this report had any documented coronary artery problems. 